\title{
Fault Detection Filter Design for LTI System with Time Delays
}

\author{
Maiying Zhong ${ }^{1} \quad$ Steven X. Ding ${ }^{2} \quad$ James Lam $^{3} \quad$ Chenghui Zhang ${ }^{1}$ \\ ${ }^{1}$ Control Science and Engineering School, Shandong University \\ 73 Jingshi Road, 250061 Jinan, China \\ Email: myzhong@sdu.edu.cn \\ ${ }^{2}$ Gerhard-Mercator-University Duisburg, Germany \\ Email: s.x.ding@uni-duisburg.de \\ ${ }^{3}$ Department of Mechanical Engineering, The University of Hong Kong \\ Email: jlam@hku.hk
}

\begin{abstract}
This paper deals with the fault detection filter design problem for linear time invariant time-delay systems with unknown input. The core of our study is to a) take the behavior of delayed state and measurement into consideration when the observer-based fault detection filter is constructed; b) solve the formulated fault detection filter design problem by combining of using the left eigenstructure assignment approach and $H_{\infty}$ optimization technique. Through a suitable choice of the filter gain matrices and residual weighting matrix, the residual can be completely decoupled from the delay-free unknown input, while the influence of the delayed unknown input on residual is minimized in the sense of $H_{\infty}$ norm. Numerical simulation is used to illustrate the efficiency of the proposed method.
\end{abstract}

Key words. Eigenstructure assignment, fault detection, filter, $H_{\infty}$ optimization, time delay

\section{Introduction}

In the past three decades, many significant results concerning fault detection and isolation (FDI) problems have been developed, see e.g $[1,3,4,10,11]$ and references therein. However, most of the achievements are for delay-free systems. Although, time delay is an inherent characteristic of many physical systems, such as rolling mills, chemical processes, water resources, biological, economic and traffic control systems, only few researches on FDI have been carried out for them $[2,5,6,7,9]$. Note that Jean-Yves and Woihida [5] study only the fault isolation problem for a kind of discrete-time system; Jiang et al. [6] deal with the nominal case fault identification (without considering the influence of model uncertainty and unknown in- puts); Liu and Frank [9] formulate the fault detection filter (FDF) design problem as a two-objective nonlinear programing problem where no analytic solution can be constructed in general; Jiang et al. [7] extends the results in [9] to the discrete-time case. The authors' earlier study in [2] has also developed an FDF design approach based on $H_{\infty}$-filtering, but the most important and difficult issue concerning the selection of a so-called reference residual model has not been successfully solved. An efficient way to tackle the fault detection problem for time-delay systems is as yet to be developed.

The main focus of this paper is to deal with the FDF design problem for LTI time-delay systems with unknown input. An FDF will be developed such that the influence of unknown input on residual is to be minimized to increase the robustness to unknown input, while the effect of fault is to be maximized to enhance the sensitivity to fault. The basic idea of our study is first to construct a new form of observer-based FDF and, based on which, to decompose the generated residual into three parts: the effect of delay-free unknown input, the influence of delayed unknown input and past information of the fault, the contribution of current faults on the residual. Then, by decoupling the residual from the delay-free unknown input and at the same time minimizing the influence of the delayed unknown input on it, to achieve perfect performance of FDI. By a combination of two often used FDI schemes, that is, eigenstructure assignment approach and $H_{\infty}$ optimization techniques, a new FDF design method for time-delay system is developed in this paper. 


\section{Problem Formulation}

We focus our attention on the FDF design problems for LTI time-delay systems, which are governed by

$$
\begin{aligned}
\dot{x}(t)= & A x(t)+\sum_{i=1}^{N} A_{i} x\left(t-\tau_{i}\right)+B u(t) \\
& +B_{f} f(t)+B_{d} d(t) \\
y(t)= & C x(t)+D u(t)+D_{f} f(t) \\
x(t)= & 0(t \leqslant 0)
\end{aligned}
$$

where $x \in \mathbb{R}^{n}$ is the state vector, $u \in \mathbb{R}^{p}$ the control input vector, $y \in \mathbb{R}^{q}$ the measurement output vector, $d \in \mathbb{R}^{m}$ the unknown input vector, $f \in \mathbb{R}^{l}$ the fault to be detected and isolated. $A, B, C, D, B_{f}, B_{d}, D_{f}, D_{d}$ are known matrices with appropriate dimensions. It is assumed that system (1)-(2) is asymptotically stable; $d$ is $L_{2}$-norm bounded; fault is detectable; $\tau_{i}>0(i=$ $1,2, \ldots, N)$ denote the constant time delays; $A_{i}$ are known matrices with appropriate dimensions.

In this paper, the following observer-based FDF is proposed

$$
\begin{aligned}
\dot{\hat{x}}(t)= & A \hat{x}(t)+\sum_{i=1}^{N} A_{i} \hat{x}\left(t-\tau_{i}\right)+B u(t)+H(y(t) \\
& -\hat{y}(t))+\sum_{i=1}^{N} H_{i}\left(y\left(t-\tau_{i}\right)-\hat{y}\left(t-\tau_{i}\right)\right) \\
\hat{y}(t)= & C \hat{x}(t)+D u(t) \\
r(t)= & V(y(t)-\hat{y}(t))
\end{aligned}
$$

where $\hat{x} \in \mathbb{R}^{n}$ and $\hat{y} \in \mathbb{R}^{q}$ are state and measurement estimation vector respectively. $r$ is the generated residual. Filter gain matrices $H, H_{i} \in \mathbb{R}^{n \times q}$ $(i=1,2, \ldots, N)$ and weighting matrix $V \in \mathbb{R}^{p_{v} \times q}$ are parameters to be designed.

Denote $e_{x}(t)=x(t)-\hat{x}(t)$, then the dynamics of the FDF for LTI time-delay systems (1)-(2) can be expressed as

$$
\begin{aligned}
\dot{e}_{x}(t)= & (A-H C) e_{x}(t)+\sum_{i=1}^{N}\left(A_{i}-H_{i} C\right) e_{x}\left(t-\tau_{i}\right) \\
& +B_{d} d(t)+\left(B_{f}-H D_{f}\right) f(t) \\
& -\sum_{i=1}^{N} H_{i} D_{f} f\left(t-\tau_{i}\right) \\
r(t)= & V\left(C e_{x}(t)+D_{f} f(t)\right)
\end{aligned}
$$

or the following transfer function form

$$
r(s)=r_{d}(s)+r_{f}(s)
$$

where

$r_{d}(s)=V C\left(s I-A+H C-\sum_{i=1}^{N}\left(A_{i}-H_{i} C\right) e^{-s \tau_{i}}\right)^{-1}$

$$
\begin{aligned}
& \times B_{d} d(s) \\
& r_{f}(s)=V\left(C\left(s I-A+H C-\sum_{i=1}^{N}\left(A_{i}-H_{i} C\right) e^{-s \tau_{i}}\right)^{-1}\right. \\
& \times\left(\left(B_{f}-H D_{f}\right)+D_{f}-\sum_{i=1}^{N} H_{i} D_{f} e^{-s \tau_{i}}\right) f(s)
\end{aligned}
$$

The main tasks of ideal FD system design is to select $H, H_{i}(i=1,2, \ldots, N)$ and $V$ such that residual generator $(6)-(7)$ is asymptotically stable, and the generated residual satisfies

$$
\begin{array}{ll}
r(s)=0, & \text { if } f(s)=0 \\
r(s) \neq 0, & \text { if } f(s) \neq 0
\end{array}
$$

Obviously, for the $L_{2}$-norm bounded unknown input $d$, a necessary condition to achieve (11)-(12) is

$$
\begin{aligned}
& V C\left(s I-A+H C-\sum_{i=1}^{N}\left(A_{i}-H_{i} C\right) e^{-s \tau_{i}}\right)^{-1} B_{d}=0 \\
& V\left(C\left(s I-A+H C-\sum_{i=1}^{N}\left(A_{i}-H_{i} C\right) e^{-s \tau_{i}}\right)^{-1}\right. \\
& \times\left(\left(B_{f}-H D_{f}\right)+D_{f}-\sum_{i=1}^{N} H_{i} D_{f} e^{-s \tau_{i}}\right) f(s) \neq 0
\end{aligned}
$$

However, the existence conditions for suitable matrices $H, H_{i}$ and $V$ to satisfy (13)-(14) are usually too strong to be satisfied. This paper focuses on the study of FD in which totally decoupling of $r$ from $d$ may be impossible. Hence, the main task in an FDF design consists of a trade-off between maximizing the influence of $r_{f}$ on $r$ to increase the sensitivity to fault and minimizing that of $r_{d}$ to enhance the robustness to unknown input by selecting $H, H_{i}$ and $V$ apppropriately.

\section{Design of Fault Detection Filter}

First, re-write the FDF transfer function expression in (8) into the following form

$$
\begin{aligned}
r(s)= & G_{r d}(s) d(s)+\sum_{i=1}^{N}\left(G_{r \tau_{i}}(s) G_{e_{x} d}(s) d(s)\right. \\
& \left.+\left(G_{e_{x} f}(s)-G_{r f \tau_{i}}(s)\right) f(s)\right) e^{-s \tau_{i}} \\
& +G_{r f}(s) f(s)
\end{aligned}
$$

where

$$
\begin{aligned}
G_{r d}(s) & =V C(s I-A+H C)^{-1} B_{d} \\
G_{r \tau_{i}}(s) & =V C(s I-A+H C)^{-1}\left(A_{i}-H_{i} C\right)
\end{aligned}
$$




$$
\begin{aligned}
G_{r f}(s)= & V\left(C(s I-A+H C)^{-1}\left(B_{f}-H D_{f}\right)+D_{f}\right) \\
G_{r f \tau_{i}}(s)= & V C(s I-A+H C)^{-1} H_{i} D_{f} \\
G_{e_{x} f}(s)= & \left(s I-A+H C-\sum_{i=1}^{N}\left(A_{i}-H_{i} C\right) e^{-s \tau_{i}}\right)^{-1} \\
& \times\left(B_{f}-H D_{f}\right)+D_{f}-\sum_{i=1}^{N} H_{i} D_{f} e^{-s \tau_{i}} \\
G_{e_{x} d}(s)= & \left(s I-A+H C-\sum_{i=1}^{N}\left(A_{i}-H_{i} C\right) e^{-s \tau_{i}}\right)^{-1} \\
& \times B_{d} e^{-s \tau_{i}}
\end{aligned}
$$

Notice that the above generated residual is linearly divided into three parts: the effects of delay-free unknown input $G_{r d}(s) d(s)$; the influence of delayed unknown input and fault

$$
\begin{gathered}
\sum_{i=1}^{N}\left(G_{r \tau_{i}}(s) G_{e_{x} d}(s) d(s)+\left(G_{e_{x} f}(s)\right.\right. \\
\left.\left.-G_{r f \tau_{i}}(s)\right) f(s)\right) e^{-s \tau_{i}}
\end{gathered}
$$

and the main contribution of fault on residual $G_{r f}(s) f(s)$. This paper formulates the FDF design problems as finding suitable filter gain matrices $H, H_{i}$ $(i=1,2, \ldots, N)$ and weighting matrix $V$ such that

- first, to null the entries in the transfer function matrix between the residual and the delay-free unknown input, that is, to satisfy

$$
G_{r d}(s)=V C(s I-A+H C)^{-1} B_{d}=0
$$

by suitably selecting $H$ and $V$, which can be handled by applying eigenstructure assignment approach. While the other requirements, such as the sensitivity of residual to fault, the influence attenuation of delayed unknown input as well the FDF stability, can be guaranteed by using the design freedom of $H$ and $V$ as well as the furthermore designing of $H_{i}(i=1,2, \ldots, N)$.

- based on $H$ and $V$ obtained in first step, to find $H_{i}$ such that system (6)-(7) is asymptotically stable and the influence of delayed unknown input is minimized. It can be achieved by minimizing the $H_{\infty}$ norm of transfer function from unknown input $d$ to $\sum_{i=1}^{N} G_{r \tau_{i}}(s) e^{-s \tau_{i}} e_{x}(s)$, that is,

$$
\min _{H_{i}} J
$$

where

$$
J=\left\|\sum_{i=1}^{N} G_{r r_{i}}(s) G_{e_{x} d}(s)\right\|_{\infty}
$$

or loosely by making $\gamma(>0)$ small in terms of the feasibility of

$$
\left\|\sum_{i=1}^{N} G_{r \tau_{i}}(s) G_{e_{x} d}(s)\right\|_{\infty}<\gamma
$$

Remark 1 The core of our study is first to divide the residual $r$ linearly into three parts and, based on this, to design the FDF by decoupling the residual from the delay-free unknown input, while the influence of the delayed unknown input is minimized under the $H_{\infty}$ norm. The sensitivity of the residual to fault can be enhanced by using the design freedom in $H$ and $V$. Our proposed design scheme is a combination of two most commonly used FDI schemes - eigenstructure assignment approach and $H_{\infty}$ optimization technique.

\section{Main Results}

\subsection{Delay-free Unknown Input Decoupling}

One of the most important unknown input decoupling FDI approach is the eigenstructure assignment, in which some left eigenvectors of the observer are assigned to be orthogonal to the unknown input distribution directions. In this way, the residual can be made robust against the unknown input [11]. For our purpose, the following results of left eigenstructure assignment in [11] are used to determine $H$ and $V$.

Lemma 1 [11] A necessary condition to satisfy (16) is that there exists a matrix $V$ to achieve

$$
V C B_{d}=0
$$

Lemma 2 [11] The sufficient conditions for satisfying the unknown input de-coupling requirement (16) are

(1) $V C B_{d}=0$.

(2) All rows of the matrix $V C$ are left eigenvectors of $(A-H C)$ corresponding to any eigenvalues.

Just as mentioned in [11], $V$ can be determined based on Lemma 1, while the independent row number of $V$ is normally chosen as

$$
p_{v}=q-\operatorname{rank}\left(C B_{d}\right) \leqslant q
$$

Lemma 2 indicates that the row of $V C$ can be used as the $p_{v}$ left eigenvectors of $(A-H C)$ corresponding to some eigenvalues, which are chosen according to the desired dynamic property of the residual. The remaining $\left(n-p_{v}\right)$ left eigenvectors can be chosen so that the effect of the most important factor of fault $G_{r f}(s) f(s)$, is maximized. For the detailed principles, the solvability condition and the design procedures of $V$ and $H$, interested readers are referred to [11]. 
4.2 $H_{i}$ Design via $H_{\infty}$ Optimization

With the above obtained matrices $H$ and $V$, residual generator (15) becomes

$$
\begin{aligned}
r(s)= & \sum_{i=1}^{N}\left(G_{r \tau_{i}}(s) e_{x}(s)-G_{r f \tau_{i}}(s) f(s)\right) e^{-s \tau_{i}} \\
& +G_{r f}(s) f(s)
\end{aligned}
$$

The remaining task of FDF design for LTI time-delay systems (1)-(2) is to determine matrices $H_{i}$ such that the residual generator is asymptotically stable and the requirement (17) or (18) is satisfied. Denote

$$
r_{e}(s)=\sum_{i=1}^{N}\left(G_{r \tau_{i}}(s) e_{x}(s)-G_{r f \tau_{i}}(s) f(s)\right) e^{-s \tau_{i}}
$$

which can also be expressed as

$$
\begin{aligned}
\dot{e}_{x}(t)= & (A-H C) e_{x}(t)+\sum_{i=1}^{N}\left(A_{i}-H_{i} C\right) e_{x}\left(t-\tau_{i}\right) \\
& +B_{d} d(t)+\left(B_{f}-H D_{f}\right) f(t) \\
& -\sum_{i=1}^{N} H_{i} D_{f} f\left(t-\tau_{i}\right) \\
\dot{\xi}(t)= & (A-H C) \xi(t)-\sum_{i=1}^{N} H_{i} D_{f} f\left(t-\tau_{i}\right) \\
& +\sum_{i=1}^{N}\left(A_{i}-H_{i} C\right) \dot{e}_{x}\left(t-\tau_{i}\right) \\
r_{e}(t)= & V C \xi(t)
\end{aligned}
$$

where $\xi(t) \in \mathbb{R}^{n}$ is an auxiliary state vector. Then matrices $H_{i}$ are determined according to find $H_{i}$ that system (21)-(23) is asymptotically stable and satisfies

$$
\left\|G_{r_{e} d}(s)\right\|_{\infty}<\gamma
$$

with $\gamma$ as small as possible in terms of the feasibility of (24), where

$$
\begin{aligned}
& G_{r_{e} d}(s)=\sum_{i=1}^{N} V C(s I-A+H C)^{-1}\left(A_{i}-H_{i} C\right) \\
& \times\left(s I-A+H C+\sum_{i=1}^{N}\left(A_{i}-H_{i} C\right) e^{-s \tau_{i}}\right)^{-1} B_{d} e^{-s \tau_{i}}
\end{aligned}
$$

Now we are in a position to solve $H_{i}$, the following lemma is required.

Lemma 3 [8] Given $\gamma>0$ and system model

$$
\begin{array}{r}
\dot{x}(t)=A x(t)+\sum_{i=1}^{N} A_{i} x\left(t-\tau_{i}\right)+B w(t) \\
z(t)=C x(t)+D w(t) \\
x(t)=0(t \leqslant 0)
\end{array}
$$

if there exist matrices $P>0, Q>0$ and $R_{i}>0(i=$ $1,2, \ldots, N)$ such that the $L M I$

$$
\left[\begin{array}{cccccc}
\Gamma_{1} & P A_{1} & \cdots & P A_{N} & P B & C^{T} \\
A_{1}^{T} P & -R_{1} & 0 & \cdots & \cdots & 0 \\
\vdots & 0 & \ddots & 0 & \cdots & 0 \\
A_{N}^{T} P & \vdots & 0 & -R_{N} & 0 & 0 \\
B^{T} P & \vdots & \vdots & 0 & -\gamma^{2} I & D^{T} \\
C & 0 & 0 & 0 & D & -I
\end{array}\right]<0
$$

holds, where

$$
\Gamma_{1}=A^{T} P+P A+\sum_{i=1}^{N} R_{i}
$$

then the system under consideration is asymptotically stable and

$$
\left\|G_{z w}(s)\right\|_{\infty}<\gamma
$$

For some given $\gamma>0$, the following theorem provides a sufficient solvability condition and the solution of $H_{i}$ $(i=1,2, \ldots, N)$ to ensure the asymptotic stability of (21) $-(23)$ and $H_{\infty}$ norm constraint (24).

Theorem 4 Given filter gain matrix $H$, weighting matrix $V$ and constant $\gamma>0$, if there exist matrices $P>0, \tilde{R}_{i}>0$ and $H_{i}(i=1,2, \ldots, N)$, such that the matrix inequality

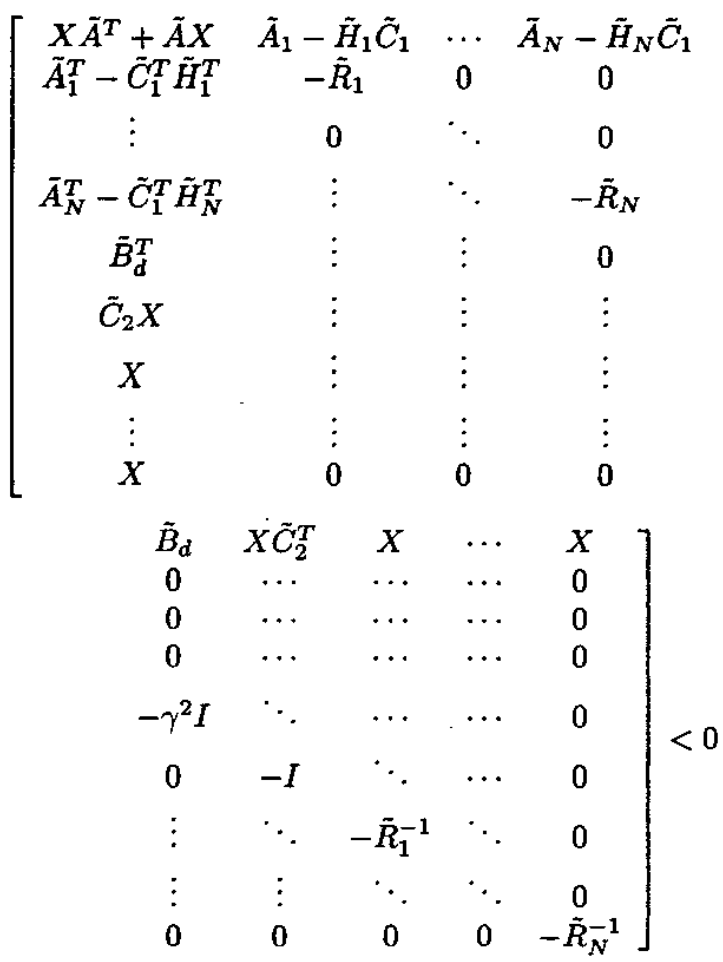


holds, then system (21)-(23) is asymptotically stable and the $H_{\infty}$ norm constraint (24) is satisfied, where

$$
\begin{array}{r}
\tilde{A}=\left[\begin{array}{cc}
A-H C & 0 \\
0 & A-H C
\end{array}\right] \\
\tilde{A}_{i}=\left[\begin{array}{cc}
A_{i} & 0 \\
A_{i} & 0
\end{array}\right], \quad \tilde{B}_{d}=\left[\begin{array}{c}
B_{d} \\
0
\end{array}\right] \\
\tilde{H}_{i}=\left[\begin{array}{c}
H_{i} \\
H_{i}
\end{array}\right], \quad \tilde{C}_{1}=\left[\begin{array}{ll}
C & 0
\end{array}\right] \\
\tilde{C}_{2}=\left[\begin{array}{ll}
0 & V C
\end{array}\right]
\end{array}
$$

Proof. In the case of $f=0$, by denoting $\tilde{A}, \tilde{A}_{i}, \tilde{B}_{d}$, $\tilde{C}_{1}, \tilde{C}_{2}$ and $\tilde{H}_{i}$ as above in (26) $-(29)$, and

$$
\Psi(t)=\left[\begin{array}{ll}
e_{x}^{T}(t) & \xi^{T}(t)
\end{array}\right]^{T}
$$

we first re-write system (21)-(23) into

$$
\begin{aligned}
\dot{\Psi}(t)= & \left.\tilde{A} \Psi(t)+\sum_{i=1}^{N}\left(\tilde{A}_{i}-\tilde{H}_{i} \tilde{C}_{1}\right) \Psi\left(t-\tau_{i}\right)\right) \\
& +\tilde{B}_{d} d(t) \\
r_{e}(t)= & \tilde{C}_{2} \Psi(t)
\end{aligned}
$$

Then, by using Lemma 3 , it is easy to show that system (30)-(31) is asymptotically stable and constraint (24) is satisfied, if there exist matrices $P>0, \tilde{R}_{i}>0$ and $H_{i}(i=1,2, \ldots, N)$, such that the following matrix inequality

$$
\left[\begin{array}{cccccc}
\tilde{\Gamma}_{1} & \tilde{\Gamma}_{11} & \ldots & \tilde{\Gamma}_{1 N} & P \tilde{B}_{d} & \tilde{C}_{2}^{T} \\
\tilde{\Gamma}_{11}^{T} & -\tilde{R}_{1} & 0 & \cdots & \cdots & 0 \\
\vdots & 0 & \ddots & 0 & \ldots & 0 \\
\tilde{\Gamma}_{1 N}^{T} & \vdots & 0 & -\tilde{R}_{N} & 0 & 0 \\
\tilde{B}_{d}^{T} P & \vdots & \vdots & 0 & -\gamma^{2} I & 0 \\
\tilde{C}_{2} & 0 & 0 & 0 & 0 & -I
\end{array}\right]<0
$$

holds, where

$$
\tilde{\Gamma}_{1}=\tilde{A}^{T} P+P \tilde{A}+\sum_{i=1}^{N} \tilde{R}_{i}, \tilde{\Gamma}_{1 i}=P\left(\tilde{A}_{i}-\tilde{H}_{i} \tilde{C}_{1}\right)
$$

By denoting $X=P^{-1}$ and via some manipulations, matrix inequality (32) can be furthermore equivalent to matrix inequality (25).

Finally, we would like to point out that:

(i) Matrix inequality (25) is not an LMI of $\tilde{R}_{i}$ due to the appearance of $\tilde{R}_{i}$ and $\tilde{R}_{i}^{-1}$;

(ii) For any given matrices $\tilde{R}_{i}>0$, however, (25) is an LMI in $X$ and $H_{i}(i=1,2, \ldots, N)$, which can be solved with the aid of some standard numerical packages; (iii) To obtain such matrices $H_{i}$ that $\gamma$ is made small in terms of the feasibility of (24), a repeatedly use of Theorem 1 is also necessary;

(iv) To satisfy the solvability condition of $H_{i}$ and ensure satisfactory FD property, the design freedom in $H$ and $V$ can be exploited.

\section{Numerical Example}

To illustrate the effectiveness of the proposed approach, a numerical example is given in this section. Consider LTI time-delay system (1)-(2) with

$$
\begin{array}{rlrl}
A & =\left[\begin{array}{lll}
0 & 3 & 4 \\
1 & 2 & 3 \\
0 & 2 & 5
\end{array}\right], & A_{1}=\left[\begin{array}{ccc}
0 & 0.1 & 0 \\
0 & 0 & 1 \\
0 & 0.5 & 1
\end{array}\right] \\
B_{f}=\left[\begin{array}{cc}
1 & 0 \\
0.5 & 0 \\
0.2 & 0
\end{array}\right], & B_{d}=\left[\begin{array}{l}
0 \\
1 \\
0
\end{array}\right] \\
C & =\left[\begin{array}{lll}
0 & 1 & 0 \\
0 & 0 & 1
\end{array}\right], & D_{f}=\left[\begin{array}{ll}
0 & 0.1 \\
0 & 0.1
\end{array}\right]
\end{array}
$$

Since the control input has no influence on the designed residual, it is not considered in this example. According to the proposed design method, the following results are obtained:

$$
\begin{aligned}
& \gamma=0.05, \quad V \doteq\left[\begin{array}{ll}
0 & 1
\end{array}\right] \\
& H=\left[\begin{array}{ll}
9 & 4 \\
7 & 3 \\
2 & 6
\end{array}\right], \quad H_{1}=\left[\begin{array}{cc}
0.1 & 0 \\
0 & 1 \\
0.5 & 1
\end{array}\right]
\end{aligned}
$$

Suppose the unknown input $d$ is band-limited white noise with unit power given in Fig. 1. Two fault signals $f_{1}$ and $f_{2}$ are simulated with unit amplitude over time interval $[5,10] \mathrm{sec}$. Fig. 2 and Fig. 3 respectively show the generated residual signal $r(t)$ for $f=\left[\begin{array}{ll}f_{1} & 0\end{array}\right]^{T}$ and $f=\left[\begin{array}{ll}0 & f_{2}\end{array}\right]^{T}$. It can be seen that the generated residual shows good performance despite the unknown input.

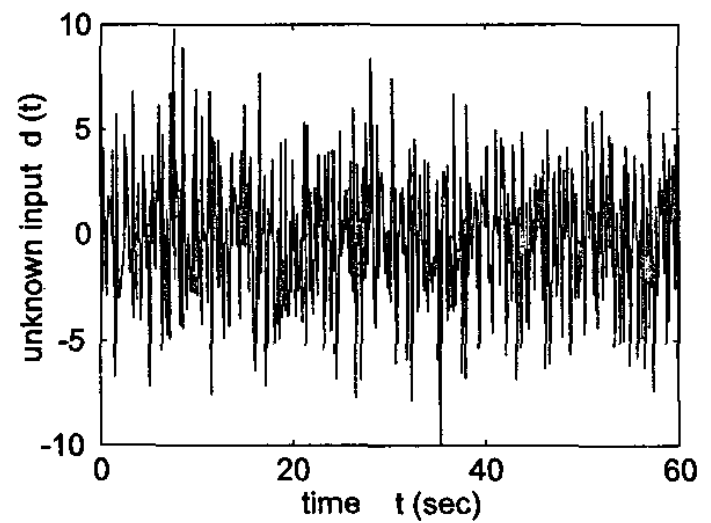

Fig. 1. Unknown input $d(t)$ 


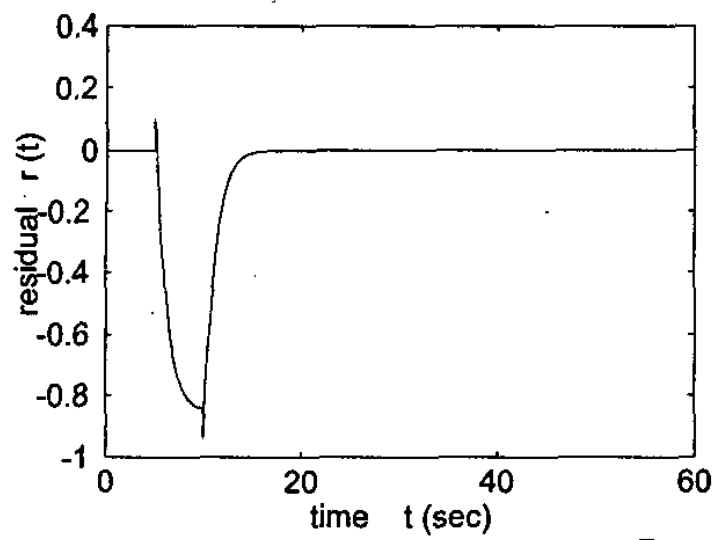

Fig. 2. Residual $r(t)$ with $f=\left[\begin{array}{ll}f_{1} & 0\end{array}\right]^{T}$

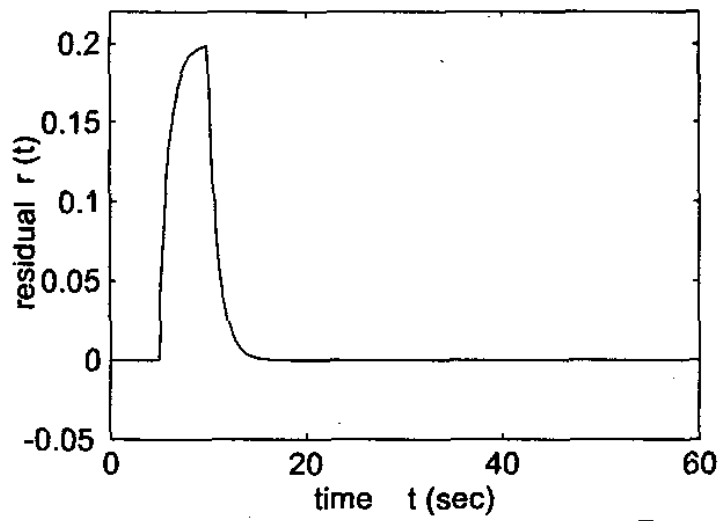

Fig 3. Residual $r(t)$ with $f=\left[\begin{array}{ll}0 & f_{2}\end{array}\right]^{T}$

\section{Conclusion}

In this paper, the fault detection filter design problem for LTI time delay systems with unknown input is studied. By combining use left eigenstructure assignment FDI and $H_{\infty}$ optimization techniques, a novel approach of FDF design for LTI time-delay systems with unknown input has been proposed, in which complete decoupling of residual from delay-free unknown input is achieved by applying eigenstructure assignment FDI approach, while the influence of delayed unknown input is minimized in the sense of the $H_{\infty}$ norm. A simula tion example is presented to demonstrate the achieved results.

\section{Acknowledgment}

This research was supported, in part, by grants from the Shandong Nature Science Foundation Y2002G05, Shandong University Science Foundation, and HKU CRCG 10204304/19870.

\section{References}

[1] J. Chen and R. J. Patton. Robust Model-Based Fault Diagnosis for Dynamic Systems. Kluwer Academic Publishers, Boston, 1999.

[2] S. X. Ding, M.-Y. Zhong, and B. Y. Tang. An LMI approach to the design of fault detection filter for time-delay LTI systems with unknown inputs. In Proc. Amer. Contr. Conf., pages 2137-2142, Arlington, VA, 2001.

[3] P. M. Frank, S. X. Ding, and B. Koppen-Seliger. Current developments in the theory of FDI. In Proc. SAFEPROCESS'2000, pages 16-27, Budapest, Hungary, 2000 .

[4] J. J. Gertler. Fault Detection and Diagnosis in Engineering Systems. Marcel Dekker, USA, 1998.

[5] K. Jean-Yves and A. Woihida. Robust fault isolation observer for discrete-times systems with time delay. In Proc. SAFEPROCESS'2000, pages 390-395, Budapest, Hungary, June 2000.

[6] B. Jiang, M. Staroswiecki, and V. Cocquempot. Fault identification for a class of time-delay systems. In Proc. Amer. Contr. Conf., pages 8-10, Anchorage, USA, May 2002.

[7] B. Jiang, M. Staroswiecki, and V. Cocquempot. $H_{\infty}$ fault detection filter for a class of discretetime systems with multiple time delays. In Proc. 15th IFAC World Congress, Barcelona, Spain, July 2002. CDROM.

[8] J. H. Kim and H. B. Park. $H_{\infty}$ state feedback control for generalized continuous/discrete time-delay system. Automatica, 35:1443-1451, 1999.

[9] J. H. Liu and P. M. Frank. $H_{\infty}$ detection filter design for state delayed linear systems. In Proc. 14th IFAC World Congress, pages 229-233, 1999.

[10] R. S. Mangoubi and A. M. Edelmayer. Model based fault detection: the optimal past, the robust present and a few thoughts on the future. In Proc. SAFEPROCESS'2000, pages 64-75, Budapest, Hungary, 2000.

[11] R. J. Patton, P. M. Frank, and R. N. Clark. Issues of Fault Diagnosis for Dynamic Systems. Springer-Verlag, London Ltd, 2000. 